\title{
The Far Infrared Spectroscopic Explorer (FIRSPEX): probing the lifecycle of the ISM in the universe
}

D. Rigopoulou, M. Caldwell, B. Ellison, C. Pearson, E. Caux, et al.

D. Rigopoulou, M. Caldwell, B. Ellison, C. Pearson, E. Caux, A. Cooray, J. D. Gallego, M. Gerin, J. R. Goicoechea, P. Goldsmith, C. Kramer, D. C. Lis, S. Molinari, V. Ossenkopf-Okada, G. Savini, B. K. Tan, X. Tielens, S. Viti, M. Wiedner, G. Yassin, "The Far Infrared Spectroscopic Explorer (FIRSPEX): probing the lifecycle of the ISM in the universe," Proc. SPIE 9904, Space Telescopes and Instrumentation 2016: Optical, Infrared, and Millimeter Wave, 99042K (29 July 2016); doi: 10.1117/12.2233593

Event: SPIE Astronomical Telescopes + Instrumentation, 2016, Edinburgh, United Kingdom 


\title{
The Far Infrared Spectroscopic Explorer (FIRSPEX): Probing the lifecycle of the ISM in the Universe
}

D. Rigopoulou ${ }^{1}$, M. Caldwell ${ }^{2}$, B. Ellison ${ }^{2}$, C. Pearson ${ }^{2}$, E. Caux ${ }^{3}$, A. Cooray ${ }^{4}$, J.D. Gallego ${ }^{5}$, M. Gerin ${ }^{6}$, J. R. Goicoechea ${ }^{7}$, P. Goldsmith ${ }^{8}$, C. Kramer ${ }^{9}$, D.C. Lis ${ }^{10}$, S. Molinari ${ }^{11}$, V. Ossenkopf-

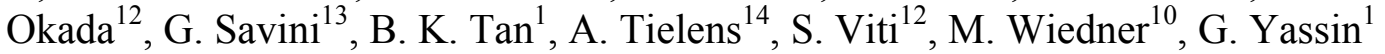

${ }^{1}$ Department of Physics, University of Oxford, Keble Road, Oxford, OX1 3RH, UK

${ }^{2}$ RAL Space, Science Technology Facilities Council, Harwell Campus, Didcot, OX11 0QX, UK

${ }^{3}$ Institut de Recherche en Astrophysique et Planétologie (IRAP formerly CESR), BP 44346, 31028

Toulouse Cedex 4, France

${ }^{4}$ Center for Cosmology, Department of Physics and Astronomy, University of California, Irvine, CA 92697, USA

${ }^{5}$ Centro Astronómico de Yebes Observatorio Astronómico Nacional Apdo. 148, 19080 Guadalajara, Spain

${ }^{6}$ LERMA, Observatoire de Paris, PSL Research University, CNRS, Sorbonne Universités, UPMC Univ. Paris 06, École normale supérieure, F-75005, Paris, France

${ }^{7}$ Grupo de Astrofisica Molecular, Instituto de Ciencia de Materiales de Madrid (CSIC) E-28049. Madrid, Spain

${ }^{8}$ Jet Propulsion Laboratory, California Institute of Technology, 4800 Oak Grove Drive, Pasadena, CA 91109, USA

${ }^{9}$ Instituto Radioastronomia Milimetrica (IRAM), Av. Divina Pastora 7, Nucleo Central, 18012, Granada, Spain

${ }^{10}$ LERMA, Observatoire de Paris, PSL Research University, CNRS, Sorbonne Universités, UPMC Univ. Paris 06, F-75014, Paris, France

${ }^{11}$ INAF-Istituto di Astrofisica e Planetologia Spaziale, via Fosso del Cavaliere 100, 00133, Roma, Italy

${ }^{12}$ I. Physikalisches Institut, Universität zu Köln, Zülpicher Str. 77, 50937 Köln, Germany

${ }^{13}$ Department of Physics and Astronomy, UCL, Gower St., London, WC1E 6BT, UK

${ }^{14}$ Leiden Observatory, Leiden University, PO Box 9513, Leiden, The Netherlands

\begin{abstract}
The Far Infrared Spectroscopic Explorer (FIRSPEX) is a novel European-led astronomy mission concept developed to enable large area ultra high spectroscopic resolution surveys in the THz regime. FIRSPEX opens up a relatively unexplored spectral and spatial parameter space that will produce an enormously significant scientific legacy by focusing on the properties of the multi-phase ISM, the assembly of molecular clouds in our Galaxy and the onset of star formation; topics which are fundamental to our understanding of galaxy evolution. The mission uses a heterodyne instrument and a $\sim 1.2 \mathrm{~m}$ primary antenna to scan large areas of the sky in a number of discreet spectroscopic channels from L2. The FIRSPEX bands centered at [CI] $809 \mathrm{GHz},[\mathrm{NII}] 1460 \mathrm{GHz},[\mathrm{CII}] 1900 \mathrm{GHz}$ and [OI]4700 GHz have been carefully selected to target key atomic and ionic fine structure transitions difficult or impossible to access from the ground but fundamental to the study of the multi-phase ISM in the Universe. The need for state-of-the-art sensitivity dictates the use of superconducting mixers configured either as tunnel junctions or hot electron bolometers. This technology requires cooling to low temperatures, approaching $4 \mathrm{~K}$, in order to operate. The receivers will operate in double sideband configuration providing a total of 7 pixels on the sky. FIRSPEX will operate from L2 in both survey and pointed mode enabling velocity resolved spectroscopy of large areas of sky as well as targeted observations.
\end{abstract}

Keywords: Far-Infrared, millimeter, heterodyne, spectroscopy, space, astrophysics 


\section{INTRODUCTION}

Although infrared (IR) astrophysics is the youngest amongst astronomical disciplines, great strides have taken place in the last 30 years since the first IR space mission, the Infrared Astronomical Satellite (IRAS). The recent advent of the Herschel Space Observatory [1] demonstrated the immense power of Far-Infrared (hereafter FIR) observations in exploring the Dusty Universe. The superb photometric capabilities of Herschel have afforded panoramic views of large areas of the Galaxy by imaging the FIR dust emission (e.g. [2],[3],[4]). Large area extragalactic surveys have uncovered thousands of galaxies (e.g. [5]). Herschel spectroscopic observations made the first inroads into disentangling the complex physics of the ionized, atomic and molecular gas. The complex processes that initiate cloud collapse enabling the onset of star formation and subsequent stellar evolution leave their imprint on the Interstellar Medium (ISM) of our Galaxy and that of external galaxies. By studying the phase structure of the ISM we can begin to unravel the processes that control the heating and cooling of the clouds that eventually regulate star formation.

The [CII] $158 \mu \mathrm{m}$ line is usually the brightest emission line in the ISM and, together with [OI] 63/146um [NII] $122 / 205 \mu \mathrm{m}$ and the [OIII] $52 / 88 \mu \mathrm{m}$, can be used to trace the physical conditions in diffuse clouds and the extended component of giant molecular clouds (GMCs, [6]). However, only high-spectral resolution (velocity-resolved) observations are capable of disentangling the emission from the different components prevalent in the ISM (e.g.) enabling us to study their dynamical properties. Such observations have been extremely limited (especially for largescale mapping). In addition, due to the attenuating effect of the Earth's atmosphere these key transitions are not easily accessible from the ground, and high-altitude balloon and aircraft platforms suffer from residual atmospheric attenuation at FIR wavelengths, but more importantly, spatial coverage.

Thus, there exists a need for a satellite containing a dedicated high spectral resolution terahertz (THz) payload for astronomical research. The Far-Infrared Spectroscopic Explorer (FIRSPEX) concept fulfills this scientific requirement. It comprises four heterodyne detection bands targeting key transitions in the $\mathrm{THz}$ frequency range. The FIRSPEX instrument payload concept builds upon and benefits from European technological heritage whilst paving the way for new discoveries in the relatively unexploited domain of large area, velocity resolved surveys. Observations in the four FIRSPEX bands have been designed to serve three intertwined science themes. Firstly, a fully sampled velocity resolved map of the Galactic Plane ( $\sim 3600$ sq. deg) targets the physical and dynamical properties of the ISM in our Galaxy. Secondly mapping observations of a volume-limited sample of nearby galaxies will establish the role of environment on the ISM, and how the properties of the ISM affect star formation. Thirdly, a deep blind FIR spectroscopic survey ( $\sim 400$ sq. deg.) will probe the physics of distant galaxies and establish the properties of the gas and how it impacts on galaxy evolution from "cosmic noon" to the galaxies we see today. In what follows, we briefly summarize the main scientific drivers of the FIRSPEX mission and outline the design of the payload.

\section{SCIENCE DRIVERS FOR THE FIRSPEX MISSION}

\subsection{The Lifecycle of the ISM in our Galaxy}

Herschel's muti-wavelength observations of extended emission from the diffuse ISM and dense filamentary structures (e.g. [2],[3]) have offered unparalleled views of the Galactic Plane. The Hi-GAL survey is delivering a transformational view of the evolutionary path that enables cold and diffuse interstellar material to condense into clouds and filaments that then fragment into protocluster-forming dense clumps. However, a similar study of the properties of the gaseous phase of the ISM is missing. 
The FIRAS instrument on the COBE satellite [9] surveyed spectroscopically the entire sky from 0.1 to $10 \mathrm{~mm}$ with a spatial resolution of $7^{\circ}$ and a velocity resolution of $1000 \mathrm{~km} / \mathrm{s}$. FIRAS determined that the $\mathrm{C}+$ line is the dominant cooling line of the ISM at $\sim 0.3 \%$ of the continuum infrared emission while the $\mathrm{N}+\& \mathrm{C}$ lines are less intense by a factor of 10 and 100, respectively. As reported by [10] all three lines peak in the central regions of our galaxy (the molecular ring) and originate from a layer with a thickness of a few degrees, but the mission had insufficient resolution to relate the emission to specific components and sources within the ISM.

The Balloon-borne Infrared Carbon Explorer (BICE, [11]) mapped some 100 square degrees around the Galactic Centre with a spatial resolution of $15^{\prime}$ and a velocity resolution of $175 \mathrm{~km} / \mathrm{s}$. The BICE results revealed that the diffuse ISM is more emissive in $\mathrm{C}+$ relative to the far-IR dust emission than compact regions of massive star formation or the Galactic Centre, further emphasizing the importance of the cold neutral medium for the total $\mathrm{C}+$ emission from the Milky Way.

The Galactic Observations of the Terahertz-C $+(\mathrm{GOTC}+)$ project (e.g.[7],[12]) targeted some 450 LOS covering $360^{\circ}$ in longitude, but not targeting specific regions and, of course, was highly under-sampled in angle. Mostly, though, with its high spectral resolution, which allowed identification of individual clouds, this survey provided a first view of the tremendous advances in our understanding of the physics of the ISM that awaits a full C+ survey of the Galactic Plane.

FIRSPEX will cover a continuous area of $3600 \mathrm{deg}^{2}$ on/around the Galactic Plane (Figure 1) in four THz bands at a spectral resolution of $\lambda / \Delta \lambda \sim 10^{6}$. The power of velocity-resolved FIR imaging-spectroscopy, will allow us to address crucial questions that will drastically improve our understanding of molecular cloud formation, the role of environment and feedback in star formation and its link to galaxy evolution. FIRSPEX observations of the Galaxy focus on the following key questions:

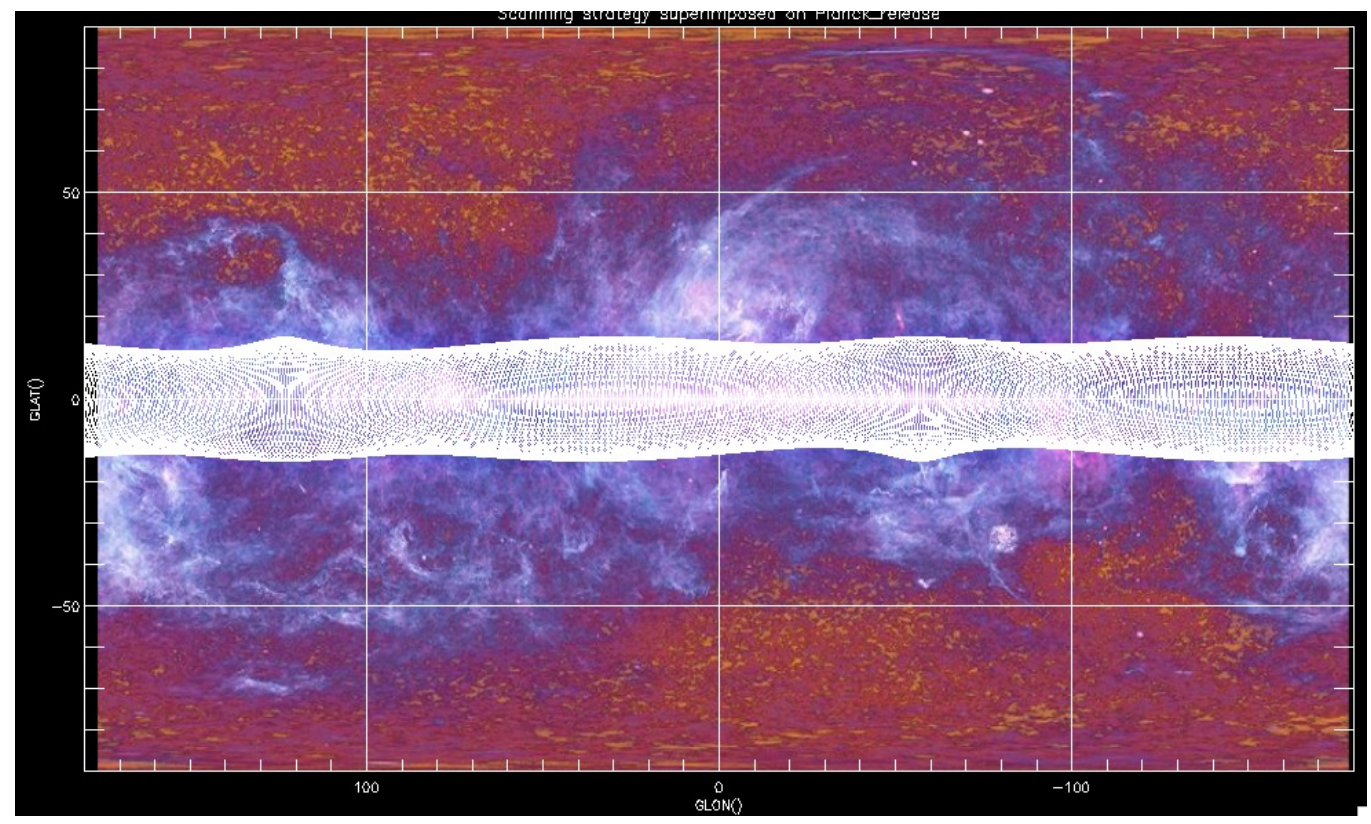

Figure 1 : The planned FIRSPEX survey of the Galactic Plane (white) superimposed on a Planck image (credit: ESA/HFI/LFI)

\section{How do molecular clouds form?}

In the Galactic plane the ISM forms molecular clouds through accretion of material onto existing denser clouds. The filamentary structures observed by Herschel support this scenario, but the accretion has not been confirmed observationally. As the accreted material undergoes a transition from atomic hydrogen to low-density molecular hydrogen and finally denser molecular gas, also visible in $\mathrm{CO}$, we need to disentangle the various phases of the ISM to probe molecular cloud formation. Resolving the velocities of the different components that determine the accretion time scales, turbulent driving and infall velocities is a pre-requisite to our understanding of star-formation on galactic scales.

\section{What fraction of the baryonic matter is in CO-dark clouds?}

High spectral resolution observations of $\mathrm{C}+$ will enable us to quantify the fraction of CO-dark gas, which is likely to 
contain a significant fraction of the baryonic matter in the Galaxy, but is invisible in most other tracers. By comparing the phase distribution with the known distribution of energetic sources in the Galaxy we can quantify the effects of the Galactic metallicity gradient and measure the global trapping efficiency of the ISM for the different heating sources.

\section{What heats the ISM?}

Radiative feedback from young stellar sources is one of the main heating sources for the Galactic ISM. FIRSPEX observations of the two main cooling lines of the ISM, [CII] and [OI], will provide a complete census of the UV heating of the Galactic ISM from UV radiation in terms of photon-dominated regions (PDRs) and mechanical energy injection via shocks and outflows. This allows to globally assess the impact of stellar feedback on the evolution of the ISM structure and it allows us to "calibrate" the contribution of the different phases to the integrated [OI], [NII], and [CII] emission observed in other galaxies.

\subsection{The ISM in Nearby Galaxies}

Some of the fundamental science questions in the study of local galaxies are: What regulates star formation in galaxies? How does the ISM vary with environment? These questions have important cosmological implications and, as such, it is important to answer them in the local Universe before we attempt to understand the distant Universe. Such questions can be addressed with a detailed study of the multi-phase gas component and its properties in external galaxies of different types (starburst, ellipticals, mergers etc). These properties seem to be quite different from those seen in Galactic star forming regions and this may impact the extragalactic star formation efficiencies and rates. Existing atomic and molecular data, for at least the nearest galaxies, show a chemical diversity and complexity that cannot be explained by a one-component, steady-state chemical model, and indicates how relative abundances between atoms and molecules may be able to provide insights into the physical distribution of the gas and the energetics of these galaxies.

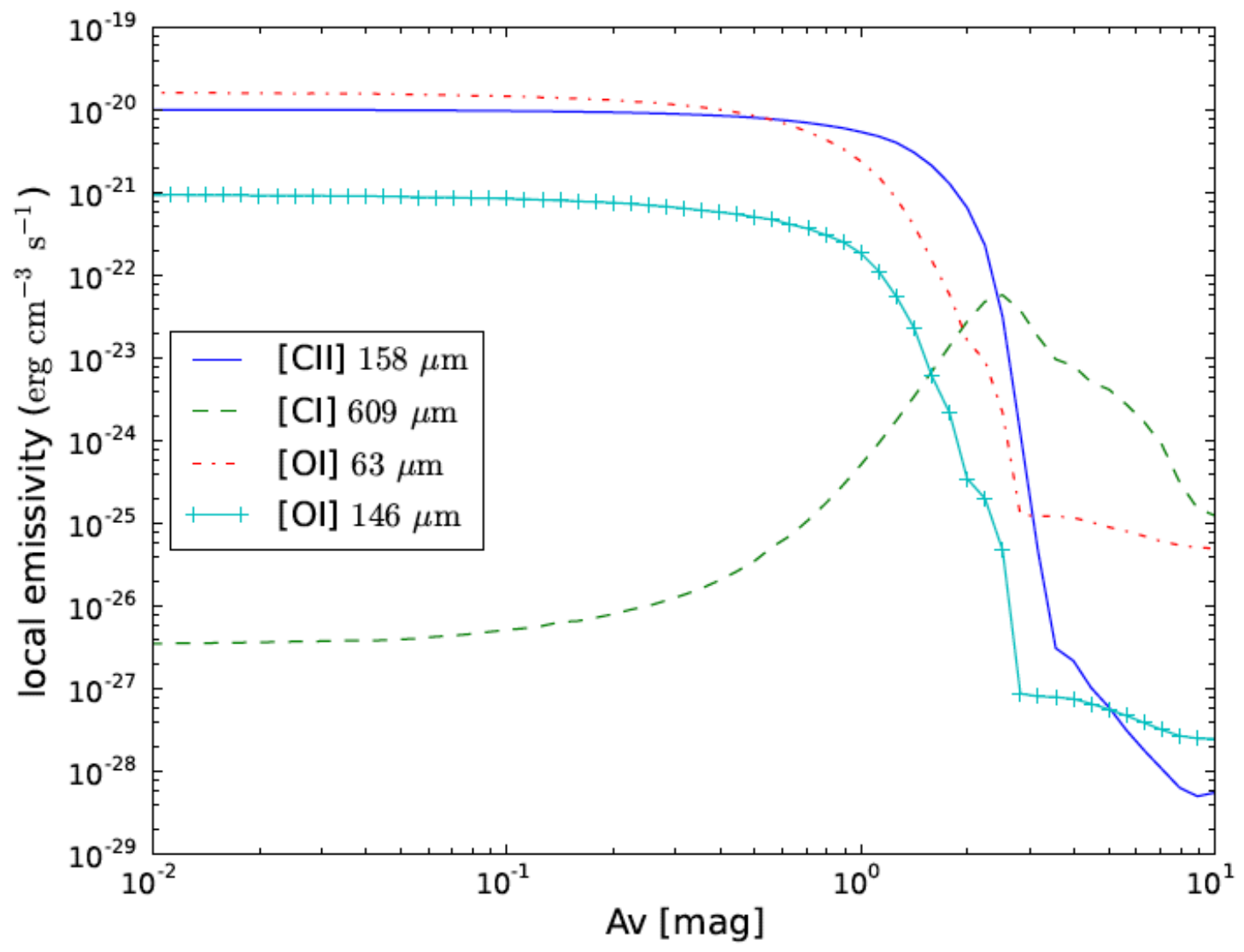

Figure 2: Local emissivities of $[\mathrm{CII}],[\mathrm{OI}] 63$, [OI]146 and $[\mathrm{CI}] 609$ microns for a PDR of uniform density of $\mathrm{n}=10^{3} \mathrm{~cm}^{-3}$ interacting with a plane-parallel UV field of strength $\chi=10^{3.5} \chi_{0}$ (from [13]). 
Observations of the most abundant gas-phase atoms and molecules in nearby galaxies allow us to study the physical characteristics of galaxy environments and measure the amount of gas in each phase of the ISM.

FIRSPEX offers the unique opportunity to obtain for the first time velocity-resolved maps of the major cooling lines of the ISM in galaxies. High spectral resolution is needed to disentangle the contributions of the various ISM phases along the same line of sight, i.e. the contributions from the dense, star forming molecular gas, photon dominated cloud interfaces, the diffuse molecular and atomic material, and the ionized gas. This will shed unprecedented light on the interplay of the ISM phases and their specific role in the cycle of matter inside galaxies, as well as in their evolution. In particular FIRSPEX observations of nearby galaxies focus on: determining the mass of CO-dark molecular gas as a function of environment, establishing the fraction of [CII] emission from the various phases of the ISM and finally, use of 3D PDR models to ascertain abundances and phase transitions of the ISM.

\subsection{The ISM of Distant Galaxies}

FIR fine structure (FS) lines provide an extremely powerful tool to probe the physical conditions of star formation in galaxies. Several studies have examined their reliability as Star Formation Rate (SFR) indicators (e.g. [14],[15],[16]). However, there is a considerable gap in redshifts between observations of FIR FS lines in relatively nearby galaxies with Herschel and detections of FIR FS lines in distant $(z>3)$ galaxies routinely reported by ALMA. If we are to establish the use of FIR FS lines as SFR indicators in the very distant Universe then it is essential to trace their evolution from present day till about redshift 3 . We need to understand the physics driving the emission of cooling lines at an epoch where the Universe was very energetic, the cosmic 'noon'.

FIRSPEX deep 'blind spectroscopic surveys' will detect galaxies based on their line emission. Using the four FIRSPEX Bands we will detect [OI], [OIII], [CII], [NII] and [NIII] in various redshifts bins in the range $0.3<\mathrm{z}<3$. The FIRSPEX deep fields are located in prime strategic fields with deep ancillary data at other wavelengths. Such observations will allow us to characterize the ISM of distant galaxies and how the properties of the gas affect star formation efficiencies and subsequent galaxy evolution.

\section{THE FIRSPEX PAYLOAD}

The scientific requirements for the FIRSPEX mission calls for a payload configuration that combines high detection sensitivity and high spectral resolving power (resolution order $>10^{6}$ ) within the Terahertz frequency domain. The sensitivity requirement is satisfied with the use of advanced heterodyne receivers based upon superconducting mixer technology used in conjunction with frequency stable local oscillators (LOs) and advanced digital sampling and analysis techniques.

The FIRSPEX payload comprises four parallel receiver channels that can operate simultaneously and therefore independently sample neighboring regions of the far-IR sky. Each receiver channel is located within the focal plane of a $1.2 \mathrm{~m}$ diameter primary antenna, and offers angular resolution of the order of 1 arcmin. In addition, each receiver channel comprises a superconducting mixer, LO chain, stages of intermediate frequency (IF) amplification followed by digital sampling and signal processing. The need for state-of-the-art sensitivity defines the use of superconducting mixers configured as tunnel junctions and hot electron bolometers. Such technology requires cooling to a low temperature, and approaching $4 \mathrm{~K}$, in order to operate. Achieving such low temperatures in space is non-trivial, but has been demonstrated by various past missions, e.g. Odin, Planck used active closed cycle coolers. Our baseline configuration proposes to passively cool the instrument to 50K (L2 orbit) and with active cooler technology providing sub-stages with necessary heat lift at $4 \mathrm{~K}$ and $15 \mathrm{~K}$. The $4 \mathrm{~K}$ stage cools the sensitive mixers and the first stages of low noise amplification. The $15 \mathrm{~K}$ and $50 \mathrm{~K}$ stages, in addition to reducing the thermal conductive and radiation load on the $4 \mathrm{~K}$ stage, also cool vital elements of the receiver chain such as the final LO stage.

A total of four receiver channels are planned with each operating in a double sideband configuration and providing a total of 7 sampling pixels on the sky. The frequency band allocations are described within Table 1 along with estimated system sensitivities. Bands 1 through 3 use two independent mixers per frequency band giving multiple pixel sampling to 
compensate for the smaller beams. Coupling to the primary quasi-optical focal plane is accomplished via relay optics comprising a series of re-imaging mirrors. For Band 1, 2 and 3, conventional harmonic frequency up-convertors provide suitable sources of LO power injected into the mixer using simple beam splitter. For band 4, the LO source is provided by a quantum cascade laser (QCL) cooled to $\sim 50 \mathrm{~K}$. Each mixer is followed by a cooled LNA and a further stage of ambient temperature amplification, while the IF final output is processed by a dedicated fast Fourier Transform spectrometer (FFTS). Each receiver system is calibrated through the use of blackbody targets of known brightness temperature that are sequentially introduced into the optical path.

Table 1: FIRSPEX receiver channel frequency, species and performance designations

\begin{tabular}{|c|c|c|c|c|c|}
\hline Designation & Frequency (THz) & Primary Species & Secondary Species & No of Pixels & $\begin{array}{c}\text { System Noise } \\
\text { (K) }\end{array}$ \\
\hline \hline Band 1 & 0.81 & CI & $\mathrm{CO}(7-6)$ & 1 & 180 \\
\hline Band 2 & 1.45 & NII & $\mathrm{SH}^{+}, \mathrm{SO}, \mathrm{CF}^{+}, \mathrm{H}_{2} \mathrm{O}^{+}$ & 2 & 350 \\
\hline Band 3 & 1.9 & CII & ${ }^{13} \mathrm{C}+$ & 2 & 500 \\
\hline Band 4 & 4.7 & OI & - & 2 & 800 \\
\hline
\end{tabular}

The spacecraft (SC) design consists of a cold Payload Module (PM) that includes the telescope and instrument box. Positioned at the Lagrangian orbit L2, the PM is located on the anti-sun side of a sunshade and using a multiple Vgroove thermal shield arrangement as per other missions at L2 (Planck, also as outlined in ESA's Next Generation Cryogenic Infra-Red Telescope study) is passively cooled to $\sim 50 \mathrm{~K}$. The cold PM provides a low temperature starting point for the closed- cycle cooler system, with use of instrument radiators, allowing the coolers to achieve the $15 \mathrm{~K}$ and $4 \mathrm{~K}$ stages. The warm Service Module (SM) is located on the sun-facing side.

The payload harnesses, and cryo-coolers for the instrument, have to penetrate through the V-groove shields, and they make use of the progressively colder stages of the shields to achieve required cooling. The cooling scheme uses a 2-stage Stirling cryo-cooler in the shields, following ESA's NG-CryoIRTel design, and cools to $\sim 15 \mathrm{~K}$. This latter stage cools a $15 \mathrm{~K}$ enclosure for the receiver and precools the Joule-Thompson cooler which provides heat lift at $\sim 4 \mathrm{~K}$. A design driver is the need to minimise the power dissipation in the cold stages ( $4 \mathrm{~K}$ and $15 \mathrm{~K}$ ), due to the limited capacity of the cryocoolers. This is accomplished through careful thermal and harnesses design.

The LO generation, receiver back-end electronics, IF components and FFTS, is mostly located within the SM. Also located within the SM are the compressors required for the Stirling and Joule-Thompson cryo-coolers, including a gaspreparation system for the latter; the cooler and on-board calibration target and target deployment electronics, and instrument control functions. A schematic of the proposed payload structure is shown in Figure 3.

This mission will be operated in two distinct modes. Mode 1 allows large area surveys around the galactic plane to be performed by continuously varying the SC pointing. Mode 2 extends the spatial sampling range to higher out-of-plane positions and allows for deep integrations on extragalactic sources.

\section{CONCLUSIONS}

We have presented a new astronomy mission concept, FIRSPEX, developed to carry out large area spectroscopic surveys of the far-IR sky at high spectral resolution. The FIRSPEX mission aims to shed new light on the assembly of molecular clouds, the onset of star formation, the CO-dark molecular gas and the properties of the multi-phase ISM all crucial parameters in understanding galaxy formation and evolution. FIRSPEX comprises a $1.2 \mathrm{~m}$ telescope and a heterodyne 
payload cooled to $4 \mathrm{~K}$ operating from L2. FIRSPEX will operate for 5 years providing a long-lasting legacy of large area spectroscopic surveys of the IR Sky.

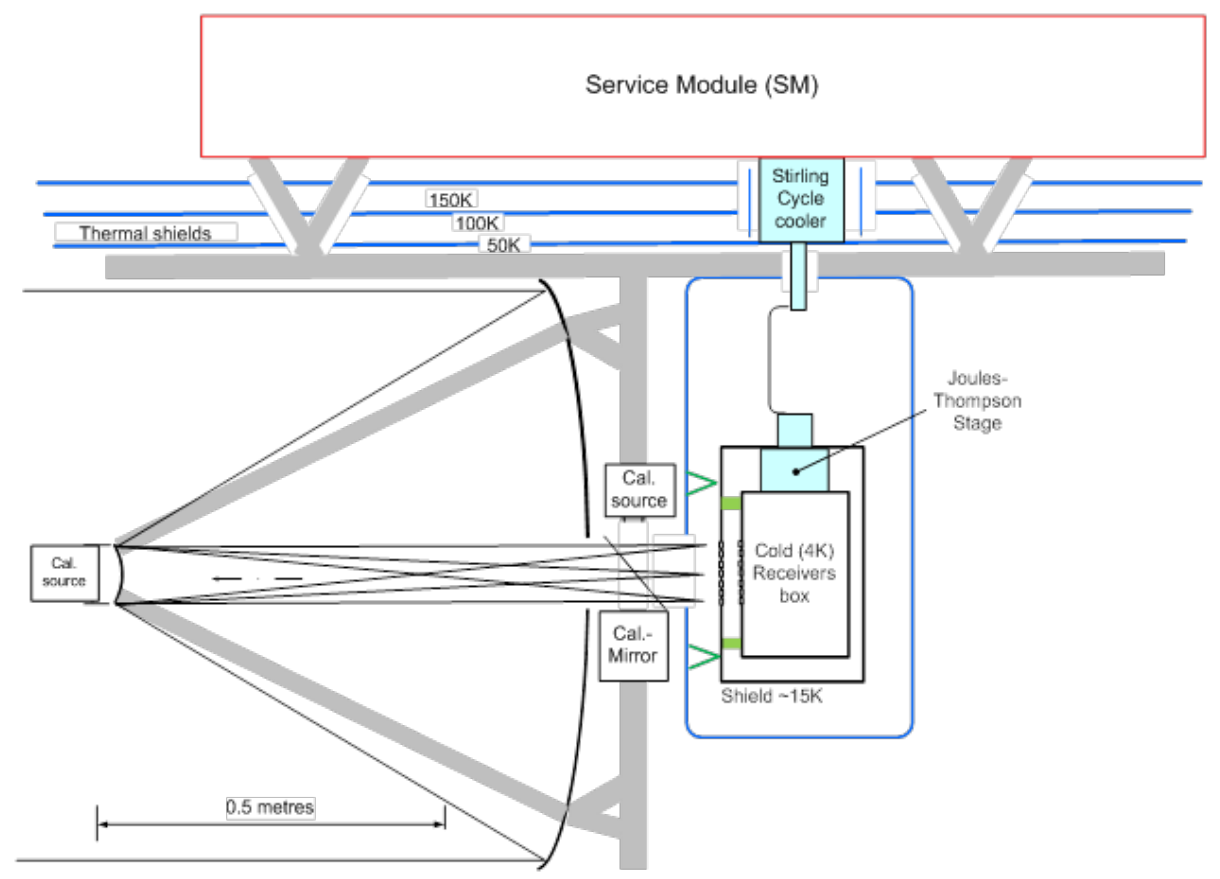

Figure 3: A schematic of the proposed FIRSPEX payload structure

\section{Acknowledgments}

Part of the work presented here has been supported by the John Fell Oxford University Press (OUP) Research Fund. This research was carried out in part at the Jet Propulsion Laboratory, California Institute of Technology, under a contract with the National Aeronautics and Space Administration.

\section{REFERENCES}

[1] Pilbratt, G., L., Riedinger, J. R., Passvogel, T., et al., 2010, A\&A 518, 1

[2] André Ph., Men'shchikov, A., Bontemps, S., et al. 2010, A\&A 518, 102

[3] Molinari S., Swinyard, B., Bally, J., et al. 2010, A\&A, 518, 100

[4] Schneider N., Güsten, R., Tremblin, P., et al. 2012, A\&A 542, 18

[5] Oliver, S., Bock, J.J., Altieri, B., et al. 2012, MNRAS, 424, 1614

[6] Hollenbach \& Tielens 1999, RvMP, 71, 173

[7] Langer, W.D.,Velusamy, T., Pineda, J. L., et al. 2010, A\&A, 521, 17

[8] Pineda J. L., Langer, W. D., Velusamy, T., et al. 2013, A\&A, 554, 103

[9] Boggess, N.W., et al., 1992, ApJ, 397, 420

[10] Fixsen, D. J., Bennett, C. L., Mather, J., 1999, ApJ, 526, 207

[11] Nakagawa, T., et al. 1998, ApJS 115, 259

[12] Velusamy T., Langer, W. D., Pineda, J. L., Goldsmith, P. F., 2012, A\&A 541, 10

[13] Bisbas T. G., Bell, T. A., Viti, S., et al. 2014, MNRAS, 443, 1111

[14] de Looze I., Fritz, J, Baes, M, et al 2014, A\&A, 571, 69

[15] Rigopoulou, D., Hopwood, R, Magdis, GE, et al, 2014, ApJLett, 781, 15

[16] Magdis, G.E., Rigopoulou, D, Hopwood, R, et al, 2014, ApJ 796, 63 